\title{
Disappearance of Trisomy +8 In A Case with Myelodysplastic Syndrome After Azacitidine Therapy Followed by Lenalidomide Treatment
}

\author{
Viktoria Varbanova ${ }^{1 *}$, Raynov $\mathbf{J}^{1}$ and Lubomir Mitev ${ }^{2}$ \\ ${ }^{1}$ Clinic of Hematology, Military Medical Academy (MMA), Bulgaria \\ ${ }^{2}$ Department of Cytogenetic and Molecular Biology, Military Medical Academy (MMA), Bulgaria \\ *Corresponding author: Viktoria Varbanova, Clinic of Hematology, Military Medical Academy (MMA), Bulgaria
}

\begin{abstract}
ARTICLE INFO
Received: 幽 November 27, 2019

Published: 櫘December 05, 2019

Citation: Viktoria Varbanova, Raynov J, Lubomir Mitev. Disappearance of Trisomy

\section{ABSTRACT}

Myelodysplastic Syndrome (MDS) is clonal hematopoietic stem cell disorders with a variety of molecular and cytogenetic abnormalities that subclassify it into different genomic subgroups with specific clinic- pathological features including prognosis and therapeutic response. The aim of this case report is to describe an unusual case with MDS in which has been observed disappearance of the clone carrying both $5 q-$ and +8 after treatment with azacitidine followed by lenalidomide administration.
\end{abstract} +8 In A Case with Myelodysplastic Syndrome After Azacitidine Therapy Followed by Lenalidomide Treatment. Biomed J Sci \& Tech Res 23(4)-2019. BJSTR. MS.ID.003927.
Keywords: Myelodysplastic Syndrome; 5q- Syndrome; Lenolidomide

Abbreviations: MDS: Myelodysplastic Syndrome; Hg: Hemoglobin; BM: Bone Marrow; PLT: Platelets; IPSS: International Prognosis Scoring System; FISH: Fluorescent in Situ Hybridization

\section{Introduction}

Myelodysplastic syndrome (MDS) is clonal hematopoietic stem cell disorders characterized clinically by ineffective hematopoiesis as a consequence of abnormalities in proliferation, differentiation, and apoptosis of hematopoietic precursors and their progeny [1] MDS is associated with a variety of molecular and cytogenetic abnormalities that subclassify it into different genomic subgroups with specific clinic- pathological features including prognosis and therapeutic response [2,3]. The most defined entity is the socalled MDS with " $5 \mathrm{q}$ - syndrome" which is characterized by isolated refractory anemia, older age, female predominance, normal or elevated platelet count, megakaryocytic dysplasia, low risk of leukemia progression and good prognosis [4]. Patients with "5 syndrome" successfully respond to treatment with lenalidomide [5-8]. In contrast, treatment of MDS with lenalidomide in cases that have $5 q$ - with additional anomalies particular +8 is less effective $[7,8]$. Here we describe an unusual case with MDS in which has been observed disappearance of the clone carrying both $5 q$ - and
+8 after treatment with azacitidine followed by lenalidomide administration.

\section{Case Report}

At Nov 2016, a 61 years old woman presented at the Clinic of Hematology with a history of etiology unknown macrocytic anemia for 1 years, hemoglobin (Hg) level $81 \mathrm{~g} / \mathrm{l}$, (WBC) $7.6 \mathrm{x}$ $10^{9} / \mathrm{l}$ and platelets (PLT) $242 \times 10^{9} / \mathrm{l}$. Screening panel disclaimed iron deficiency or other alimentary associated anemia, negative test for hemolytic anemia. Peripheral blood smear revealed absence of blast population. The bone marrow aspiration revealed hypercellular bone marrow (BM) with $2 \%$ blasts and dysplastic features of all the three cell lines, the most prominent dysplasia was found in erythroblasts presented mainly with multinuclear cell nuclei. The test for ring sideroblasts was negative. Conventional cytogenetics revealed clone with isolated trisomy of chromosome 8 in $67 \%$ of the examined cells - 47,XX,+8[16]/46,XX[8]. The diagnosis of myelodysplastic syndrome refractory cytopenia with 
multilineage dysplasia was concluded [1]. Based on these finding, the risk stratification as intermediate-1 risk group according to International prognosis scoring system (IPSS) for MDS [2] and the defined serum erythropoietin level more than $500 \mathrm{mU} /$ $\mathrm{ml}$, best supported care was accepted as treatment strategy. Eighteen months (July 2018) after start of best supported care the transfusion needs increased significantly, and disease reassessment was performed with bone marrow aspiration showing blast $4 \%$ population. Conventional cytogenetics of BM revealed the following karyotype: 47,XX,+8[24]/47,XX,del(5)(q13q33),+8[3]/46,XX[2].

The Fluorescent in Situ Hybridization (FISH) with satellite enumeration DNA probe for chromosome 8 conformed the +8 clone and the 5q- specific DNA probe demonstrated that the $5 \mathrm{q}$ anomaly is presented in $21 \%$ of the cells (100 cells examined). New therapy strategy with azacytidine was start at July 2018. After two cycles with hypomethylating agent therapy was discontinued due to severe anemia ( $\mathrm{Hb}$ nadir $51 \mathrm{~g} / \mathrm{L}$ ). At October $20182^{\text {nd }}$ with lenalidomide was started with documented erythroid response, which is still preserved, but progressive thrombocytopenia was seen. Hereof bone marrow reassessment was done October 2019 showing at conventional cytogenetics that a new clone carrying only the 5q- was arisen - 46,XX,del(5)(q13q33)[3]/46,XX[22] and metaphases with +8 or combination of +8 with $5 q$ - was not presented. FISH study with $5 \mathrm{q}$ - specific DNA probe conformed the $5 q$ - anomaly in $20 \%$ of the cells (100 cells examined) and the satellite enumeration DNA probe for chromosome 8 did not find cells with trisomy of chromosome 8.

\section{Discussion}

Trisomy 8 is a common cytogenetic abnormality associated with intermediate cytogenetic risk according to IPSS and Reverse IPSS $[2,3]$. The use of Antithymocyte Globulin (ATG) for this subgroup of MDS show response rates up to 67\%, [9]. However, Solan et al. [10] demonstrated that younger age and HLA-DR15 positivity were the most significant factors favoring response to immunosuppressive treatment (IST, ATG plus cyclosporine (CsA), or CsA alone). Based on this study IST is recommended for patients $\leq 60$ years old and $\leq 5 \%$ blast in bone marrow or those with hypocellular bone marrow, PNH clone positivity or STAT-3 mutant cytotoxic T clone positivity [11]. For all other cases with/witout del(5q) symptomatic anemia low/int -1 MDS azacytidine therapy is recommended as is in the presented clinical case. In accordance with the already known hematological and nonhematological adverse events associated with azacytidine use is the discontinuation of therapy in our patients [12]. However, the reassessment of bone marrow before starting azacytidine treatment showed new clone with del(5q) which allow us to start treatment with lenalidomide. The therapeutic effectiveness of lenalidomide in 5q- low/int-1 IPSS MDS is proved in two randomized studies where most of the patients were with isolated $\operatorname{del}(5 q)$ or $\operatorname{del}(5 q)$ plus $\geq 1$ cytogenetic abnormality $[6,7]$. Erythroid hematological improvement was achieved in approximately $60 \%$ of patients. There were no data if additional cytogenetic abnormality influences the therapeutic effect. Other studies showed that additional cytogenetic abnormality including trisomy 8 significantly decreased overall survival [8] and cytogenetic response was not achieved in patients with $5 q$ syndrome with additional aberration hereofexcept of trisomy 21 or $\mathrm{t}(1 ; 22) \mathrm{q}(21 \mathrm{p} 11.2)[6]$.

Contrary in the presented case we found complete clearance of cytogenetic clone with trisomy 8 , which is unique considering the known intermediate risk associated with +8 and lower response rate. It should be noted, however that the patients were treated also with hypomethylating agent in the course of the disease, which could influence the observed clearance of +8 MDS clone. Some data from clinical studies phase II showed synergic effect from the combination of azacytidine with lenalidomide in patients with MDS int-1/int-2/high risk IPSS [12]. The effectiveness of the same combination was also investigated in the setting of acute myeloid leukemia with contradictory results [13-15]. We suppose that the hypomethylating effect of the azacytidine had initiated genomic reorganization that have been led to the activation of proapoptotic genes and that the subsequent cytotoxic influence of the lenalidomide of the pro-apoptotic cells had resulted in eradication of the clones caring +8 .

\section{Conclusion}

The presented clinical case raises the question for consecutive therapy of azacytidine and lenalidomide in the case of low/int-1 IPSS risk MDS with del(5q) with +8 additional aberration. Lenalidomide mediated its immunomodulating effect by altering cytokine production, regulating T cell co-stimulation and augmenting the NK cell cytotoxicity. The biological mechanism of action of azacytidine is based on its hypomethylating effect and direct cytotoxic effect [12]. It could be purposed that combination or consecutive of these two different therapeutic strategies could have synergic effect in such cases $5 q$ - syndrome with additional trisomy 8 , but prospective clinical trials are necessary for conclusions.

\section{Acknowledgement}

To Nedeva A, Nikolov I, Petkova N, Kindekov I, Kuyumdzhieva $\mathrm{Y}$, Anastasova-Postadzhiyan A, Kancheva T who participated in the approvment of the therapeutic strategy and in the treatment of the presented clinical case.

\section{References}

1. Vardiman JW, Thiele J, Arber DA, Brunning RD (2009) The 2008 revision of the World Health Organization (WHO) classification of myeloid neoplasms and acute leukemia: rationale and important changes. Blood 114(5): 937-951.

2. Greenberg P, Cox C, Le Beau MM, Fenaux P (1997) International scoring system for evaluating prognosis in myelodysplastic syndromes. Blood 89(6): 2079-2088.

3. Greenberg PL, Tuechler H, Schanz J, Sanz G (2012) Revised International Prognostic Scoring System for Myelodysplastic Syndromes. Blood 120(12): 242454-2465. 
4. Giagounidis AAN, Germing U, Wainscoat IS, Boultwood J, Aul C (2004) The 5q_syndrome. Hematology 9(4): 271-277.

5. Kotla V, Goel S, Nischal S (2009) Mechanism of action of lenalidomide in hematological malignancies. J Hematol Oncol 2: 36.

6. List A, Dewald G, Bennett J (2006) Lenalidomide in the myelodysplastic syndrome with chromosome 5q deletion. N Engl J Med 355(14): 14561465.

7. Fenaux P, Giagounidis A, Selleslag D (2011) A randomized phase 3 study of lenalidomide versus placebo in RBC transfusion-dependent patients with Low-/Intermediate-1-risk myelodysplastic syndromes with del5q. Blood 118(14): 3765-3776.

8. Giagounidis AAN, GermingU, Strupp C, Hildebrandt B (2005) Prognosis of patients with del(5q)MDS and complex karyotype and the possible role of lenalidomide in this patient subgroup. Ann Hematol 84(9): 569571.

9. Sloand EM, Mainwaring L, Fuhrer M 2005) Preferential suppression of trisomy 8 compared with normal hematopoietic cell growth by autologous lymphocytes in patients with trisomy 8 myelodysplastic syndrome. Blood 106(3): 841-851.

\section{ISSN: 2574-1241}

DOI: 10.26717/BJSTR.2019.23.003927

Viktoria Varbanova. Biomed J Sci \& Tech Res

(C) This work is licensed under Creative

Submission Link: https://biomedres.us/submit-manuscript.php
10. Sloand EM, Wu CO, Greenberg P, Young N, Barrett I (2008) Factors Affecting Response and Survival in Patients with Myelodysplasia Treated with Immunosuppressive Therapy. J Clin Oncol 26(15): 2505-2511.

11. https://www.nccn.org/professionals/physician_gls/pdf/mds.pdf

12. Fenaux P, Mufti GJ, Hellström Lindberg E, Santini V, et al. (2009) Efficacy of azacitidine compared with that of conventional care regimens in the treatment of higher-risk myelodysplastic syndromes: a randomised, open-label, phase III study. Lancet Oncol 10(3): 223-232.

13. Sekeres MA, Tiu RV, Rami Komrokji R, Lancet J (2012) Phase 2 study of the lenalidomide and azacitidine combination in patients with higherrisk myelodysplastic syndromes. Blood 120(25): 4945-4951.

14. Craddock C, Slade D, Santo CD , Rachel Wheat R (2019) Combination Lenalidomide and Azacitidine: A Novel Salvage Therapy in Patients Who Relapse After Allogeneic Stem-Cell Transplantation for Acute Myeloid Leukemia. JCO 37(7): 580-588.

15. Kenealy M, Hertzberg M, Benson W, Taylor K (2019) Azacitidine with or without lenalidomide in higher risk myelodysplastic syndrome \& low blast acute myeloid leukemia. Haematologica. 104(4): 700-709.

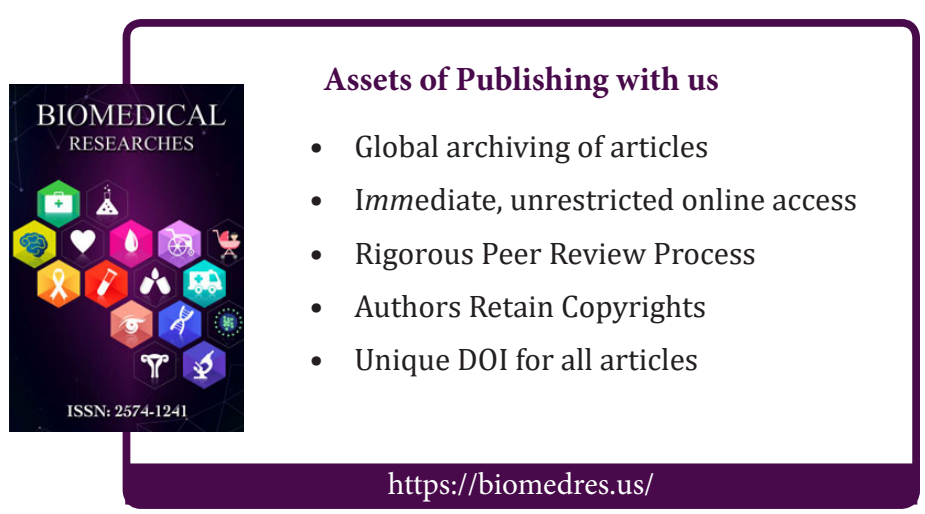

